\title{
Caries y pérdida dental en estudiantes preuniversitarios mexicanos
}

\author{
Javier de la Fuente-Hernández, M en C,(1) Marcela González de Cossío, MC, (2) Miriam Ortega-Maldonado, CD, ${ }^{(3)}$ \\ María Cristina Sifuentes-Valenzuela, M en ES.(I)
}

\section{Fuente-Hernández J, González de Cossío M, Ortega-Maldonado M, Sifuentes-Valenzuela MC. \\ Caries y pérdida dental en estudiantes preuniversitarios mexicanos. Salud Publica Mex 2008;50:235-240.}

\begin{abstract}
Resumen
Objetivos. Conocer la prevalencia de caries y pérdida dental para calcular las necesidades terapéuticas en estudiantes de educación media superior que ingresan a la Universidad $\mathrm{Na}$ cional Autónoma de México (UNAM). Material y métodos. Se realizó un estudio transversal descriptivo en el que se obtuvo una muestra de 77 I9I estudiantes correspondientes a las generaciones de ingreso al bachillerato 2003, 2004 y 2005. Los datos de salud bucal se obtuvieron a partir del índice CPOD incluido en el Examen Médico Automatizado (EMA), instrumento de autorrespuesta estandarizado que aplica la Dirección General de Servicios Médicos (DGSM). El análisis del EMA fue univariado con la finalidad de identificar la distribución y la frecuencia de las variables. Resultados. La prevalencia de caries y pérdida dental fue de 48.0 y $34.2 \%$, respectivamente, con una cuantificación del índice CPOD de 5. Las necesidades de tratamiento para caries y pérdida dental se obtuvieron en al menos un diente por estudiante. Conclusiones. Cerca de la mitad de los alumnos que ingresan al bachillerato de la UNAM requiere al menos la atención de una caries o prótesis dental. Lo anterior evidencia que las políticas en salud bucal instrumentadas no han alcanzado los índices en salud esperados en relación con los objetivos internacionales, así como la necesidad de impulsar nuevas líneas de investigación orientadas a identificar la gravedad de la enfermedad y factores vinculados con el deterioro de la salud bucal.
\end{abstract}

Palabras clave: caries dental; índice CPO; salud bucal; México
Fuente-Hernández J, González de Cossío M, Ortega-Maldonado M, Sifuentes-Valenzuela MC. Dental decay and tooth loss at the high school level in Mexican students.

Salud Publica Mex 2008;50:235-240.

\begin{abstract}
Objective. To determine the prevalence of dental decay and tooth loss, and to consider the treatment needs for students at the Mexican National Autonomous University (UNAM). Materials and Methods. A cross sectional study was designed including 77191 students corresponding to the years of entrance to high school 2003, 2004 and 2005. Oral health data were obtained from the DMF-T index included in the Automatized Medical Exam (AME), a student self-answer instrument administered by the General Medical Services Department, UNAM. The analysis of the AME was univaried with the purpose of identifying the distribution and frequency of the variables. Results. The prevalence of dental decay and tooth loss was $48.0 \%$ and $34.2 \%$, respectively. The DMF-T index was 5 . The treatment needs for dental decay and tooth loss for a student were considered as at least one lost tooth. Conclusions.Approximately half of the students from UNAM high schools need at least restorations and dental prosthesis. This suggests that oral health programs have not yet reached the expected health indexes according to international regulations, as well as the need to develop new research projects directed toward identifying disease severity and the factors that are detrimental to oral health.
\end{abstract}

Key words: decay; DMF-T index; oral health; Mexico

(I) Dirección de Odontología, Universidad Nacional Autónoma de México. Distrito Federal, México.

(2) Subdirección de Investigación y Desarrollo Humano. Universidad Nacional Autónoma de México. Distrito Federal, México.

(3) Coordinación de Odontología Preventiva y Salud Pública. Universidad Nacional Autónoma de México. Distrito Federal, México.

Fecha de recibido: 2 de mayo de 2007 Fecha de aceptado: 14 de noviembre de 2007 Solicitud de sobretiros: Mtro. Javier de la Fuente Hernández. Dirección de Odontología, Universidad Nacional Autónoma de México. Av. Universidad 3000, Circuito Escolar s/n, Edificio C, Planta baja, col. Copilco El Bajo. 04510 Del. Coyoacán, México, DF, México. Correo electrónico:fuente@servidor.unam.mx 
$\mathrm{L}$ a salud bucal se considera una parte integral del complejo craneofacial que participa en funciones vitales como la alimentación, la comunicación y el afecto; por lo tanto, la salud bucal está relacionada con el bienestar y la calidad de vida desde los puntos de vista funcional, psicosocial y económico. ${ }^{1}$

Entre las principales enfermedades que afectan la cavidad bucal figuran la caries dental, que se define como la enfermedad infecciosa de origen multifactorial caracterizada por el reblandecimiento del tejido duro del diente que evoluciona hasta formar una cavidad, o bien la pérdida de los dientes si ésta prosigue su evolución natural sin tratamiento. Gran parte de la población la padece de forma innecesaria, debido sobre todo al estilo de vida (dieta e higiene bucal inadecuada), así como al acceso restringido a los servicios de salud odontológicos, la falta de cultura de la población en cuanto al cuidado de la boca y los altos costos que la atención odontológica representa, entre otros. ${ }^{2}$

Diversos autores mencionan que la caries dental es un problema de salud pública en virtud de su elevada prevalencia. ${ }^{3-8}$ En México se ha documentado que la prevalencia de caries dental se encuentra entre $70 \mathrm{y}$ $85 \%$ en dentición secundaria a la edad de 12 años. ${ }^{9}$ No obstante las medidas de salud pública bucal instituidas (fluoración de la sal, programas nacionales de salud bucal), los problemas de morbilidad bucal ocupan un papel importante en el panorama de salud pública, toda vez que demandan conductas personales para su control más eficaz y, en buena medida, se trata de problemas que pueden prevenirse con mínimas conductas de protección a la salud. ${ }^{10}$

La Organización Mundial de la Salud (OMS), dentro de sus objetivos propuestos para el año 2000, establece para la población de 18 años la conservación de al menos 85\% de la totalidad de sus dientes, por lo que sugiere la utilización de instrumentos clinimétricos para la medición adecuada de la caries, de los cuales el más aceptado y utilizado es el índice CPOD (diente cariado, perdido y obturado), cuyo propósito fundamental es obtener información global del estado de salud bucal de una población específica mediante la sumatoria de dientes cariados, perdidos y obturados. ${ }^{11}$

De la misma manera, y en concordancia con los objetivos de salud bucal para el año 2020 recomendados por la Federación Dental Internacional (FDI), la OMS y la Asociación Internacional de Investigación Dental (IADR) plantean retos globales para los encargados de la planeación de programas en salud, en los planos nacional, regional y local; en consecuencia, se ha determinado un máximo de tres dientes cariados para la población escolar y la conservación de todos los dientes en $85 \%$ de los adolescentes de 18 años. ${ }^{12}$
Resultados de la Primera Encuesta Nacional de Caries y Fluorosis dental 1996-2000 informan que el promedio del índice CPOD a la edad de 12 años fue de 2.23; en ambas anomalías se observa un alto porcentaje de caries no tratadas. ${ }^{13}$ Asimismo, la Encuesta Nacional de Caries Dental 2001 señala que la prevalencia de caries para el grupo de edad de 15 años fue de $58 \%$ y el índice CPOD de 1.91, mientras que para el DF la prevalencia fue de $88.6 \%$ con un índice CPOD de 5.31. ${ }^{14}$

En 1993, Maupome estudió a 2596 pacientes de zonas marginales de diferentes estados de la República Mexicana y notificó un índice CPOD de 8.3 en mayores de 15 años. ${ }^{15}$ De igual modo, en el año 2000 Rivas realizó un estudió en una muestra representativa de 540 adolescentes del estado de Zacatecas y halló un CPOD de 7.2. ${ }^{16}$ Esta información es similar a la de Rivera y colaboradores del año 2006, en la que se identificó un índice CPOD de 6.8 y una prevalencia de caries de $97 \%$ en 113 estudiantes de bachillerato. ${ }^{5}$

La caries dental es un proceso que puede evolucionar y ocasionar la pérdida dental si no se atiende con oportunidad. Durante el año 2006 en la Facultad de Odontología se llevó a cabo un estudio transversal en adolescentes, en el cual se observó una prevalencia de pérdida dental de $19 \%$ y una proporción de $38 \%$ correspondió a problemas de caries dental. ${ }^{7}$ En este mismo año Jun y colaboradores, en Japón, notificaron que en pacientes de 15 a 25 años $43.3 \%$ presentó pérdida dental por razones de caries. ${ }^{8}$

Respecto de las necesidades de tratamiento, son pocos los estudios efectuados. Pese a ello, es posible referir a Pineda y colaboradores, quienes en 2000 mencionan que el porcentaje de necesidades de tratamiento para caries dental en la población escolar fue de 90 por ciento. ${ }^{19}$

Con base en los estudios revisados, la caries dental es aún el principal problema de salud pública por su elevada prevalencia. Sin embargo, como puede observarse, los estudios se enfocan en la población comprendida entre los seis y 15 años de edad, lo cual muestra una escasez de datos en individuos de mayor edad. En este contexto, el propósito del estudio fue dar a conocer de manera general la salud bucal de los adolescentes que ingresan al nivel medio superior en la UNAM; la finalidad era identificar sus necesidades de atención $y$, con ello, contribuir al establecimiento de políticas institucionales de salud bucal específicas, de tal modo que mejore la salud de los individuos y se alcancen los objetivos del milenio.

\section{Material y métodos}

El Examen Médico Automatizado (EMA) es un instrumento estandarizado de autorrespuesta que de manera 
censal se aplica a los estudiantes que ingresan a la Universidad Nacional Autónoma de México. Su propósito es conocer las condiciones de salud físicas, psicológicas y sociales..$^{20}$ Es importante señalar que el mayor porcentaje de estudiantes que ingresan a la UNAM procede del DF y su área conurbada.

El diseño del estudio fue transversal descriptivo. El universo consistió en 138931 estudiantes, de los cuales sólo 55.5\% (77 191) constituyeron la muestra de estudio, al cumplir con los siguientes criterios de inclusión: a) ingreso a nivel medio superior, b) presentar documentación para la inscripción, c) presentar valoración bucodental por parte de un profesional habilitado o participar en la exploración bucal durante la realización del EMA y d) contestar correctamente el examen. Durante la aplicación del instrumento se contó con personal capacitado del área bucal para cualquier aclaración.

Con posterioridad, las hojas se procesaron mediante un lector óptico conforme al software y codificación elaborados ex profeso mediante el programa denominado Sistema Informático de Reportes del EMA (SIREMA) con plataforma en Visualpro; este proceso permitió obtener frecuencias y porcentajes de respuesta por generación y sexo.

El protocolo se puso a consideración del Comité de Ética de la Facultad de Odontología, el cual está registrado ante la Secretaría de Salud. Este comité, con base en el Reglamento de la Ley General de Salud en Materia de Investigación en Seres Humanos, consideró que el consentimiento informado no era indispensable, toda vez que se trataba de una investigación sin riesgo. ${ }^{21}$

Para conocer la prevalencia de caries y pérdida dental se identificaron los dientes cariados y perdidos en dentición secundaria, a partir de los exámenes médicos que entregaron los alumnos al inicio del EMA. De igual manera, se hizo necesario el conteo de dientes obturados para poder calcular el índice CPOD. Las necesidades de tratamiento se reconocieron a partir de la sumatoria del número de dientes cariados dividida sobre el total del número de dientes cariados, perdidos y obturados. ${ }^{19}$

Para el análisis de la prevalencia de caries por el número de dientes afectados se consideraron límites de uno a nueve o más dientes cariados, se realizó un corte de $\leq 3$ y se tomaron como referencia los objetivos propuestos por la OMS.

\section{Resultados}

El total de la muestra quedó conformado por 77191 estudiantes de nivel medio superior. La tasa de no respuesta fue de 4.7 por ciento. La edad promedio de la población fue de 16 años 2 meses (48\% de hombres y $52 \%$ de mujeres). La prevalencia de caries fue de $48 \%$ y de pérdida dental de 23.8 por ciento. El promedio del índice CPOD fue de 5, para los tres ciclos escolares.

$\mathrm{Al}$ analizar la prevalencia de caries por año y género, no se encontraron diferencias importantes durante los tres años del estudio: $2003(29.0 \%), 2004(35.3 \%)$ y $2005(35.7 \%)$ (cuadro I).

Para el caso de la pérdida dental, en el año 2003 se observó una prevalencia menor $(31.3 \%)$ respecto de los dos años subsecuentes $(2004,34.5 \%$ y $2005,34.2 \%)$. Asimismo, los hombres registraron un porcentaje menor (2003 y 2004, 48.4\% y 2005, 47\%), en comparación con las mujeres $(2003,51.5 \%, 2004,51.6 \%$ y 2005, 53\%) (cuadro I).

En relación con el número de dientes obturados, durante los tres años del estudio los porcentajes registrados fueron similares: 2003 (29.4\%), 2004 (36.6\%) y $2005(34 \%)$ (cuadro I).

Se identificó a 36859 estudiantes con caries; de este número, con base en el punto de corte establecido $(\leq 3)$, $26491(71.9 \%)$ presentaron menos de tres dientes cariados y 10363 (28.1\%) cuatro o más dientes (figura 1).

\section{Cuadro I \\ Prevalencia de Caries, Pérdida y obturación dental en estudiantes de NIVEL Medio suPerioR, POR AÑo Y GÉNERO. MÉXICO, DF}

\begin{tabular}{|c|c|c|c|c|c|c|c|c|c|c|c|c|c|c|c|c|c|c|}
\hline \multirow[b]{3}{*}{ Condición bucal } & \multicolumn{6}{|c|}{2003} & \multicolumn{6}{|c|}{2004} & \multicolumn{6}{|c|}{2005} \\
\hline & \multicolumn{2}{|c|}{ Hombres } & \multicolumn{2}{|c|}{ Mujeres } & \multicolumn{2}{|c|}{ Total } & \multicolumn{2}{|c|}{ Hombres } & \multicolumn{2}{|c|}{ Mujeres } & \multicolumn{2}{|c|}{ Total } & \multicolumn{2}{|c|}{ Hombres } & \multicolumn{2}{|c|}{ Mujeres } & \multicolumn{2}{|c|}{ Total } \\
\hline & (n) & (\%) & (n) & $(\%)$ & (n) & (\%) & (n) & (\%) & (n) & $(\%)$ & (n) & (\%) & (n) & (\%) & (n) & $(\%)$ & (n) & (\%)) \\
\hline ental & 700 & 44.0 & 993 & 56.0 & 0693 & 29.0 & 600 & 43.1 & 7397 & 56.9 & 12997 & 35.3 & 6069 & 46.1 & 7100 & 53.9 & 13169 & 35.7 \\
\hline rdida dental & 2782 & 48.4 & 2960 & 51.5 & 5742 & 31.3 & 3072 & 48.4 & 3276 & 51.6 & 6348 & 34.5 & 2945 & 47.0 & 3332 & 53.0 & 6277 & 34.2 \\
\hline bturación dent & 4170 & 43.7 & 5372 & 56.3 & 9542 & 29.4 & 4992 & 42.0 & 6893 & 58.0 & 11885 & 36.6 & 5180 & 46.8 & 5900 & 53.2 & 11080 & 34.0 \\
\hline
\end{tabular}

Fuente: Examen Médico Automatizado. Dirección General de Servicios Médicos. Universidad Nacional Autónoma de México. Batería índice CPO. Reportes ecológicos NMS, 2003, 2004 y 2005; $n=77$ I9I estudiantes 


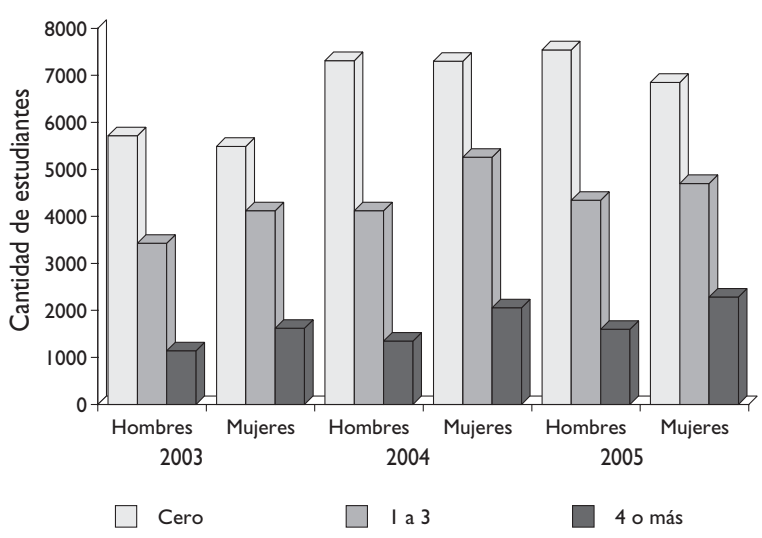

Fuente: Examen Médico Automatizado. Dirección General de Servicios Médicos. Universidad Nacional Autónoma de México. Batería índice CPO. Reportes ecológicos Nivel Medio Superior, 2003, 2004 y 2005; $n=77$ I9I estudiantes

\section{FiguRA I. DistRIBUCIÓN PORCENTUAL DE CARIES DENTAL POR} GENERACIÓN Y SEXO. MÉXICO, DF

Respecto de los 18367 estudiantes con pérdida dental, $10091(55 \%)$ presentaron pérdida de más de cuatro dientes (figura 2). De la misma manera, de los 32507 estudiantes con obturación dental, 21114 (64.9\%) tenían uno a tres dientes rehabilitados (figura 3).

La razón de dientes cariados (100 243) en cuanto al número de estudiantes (77 191) fue de 1:1, resultado

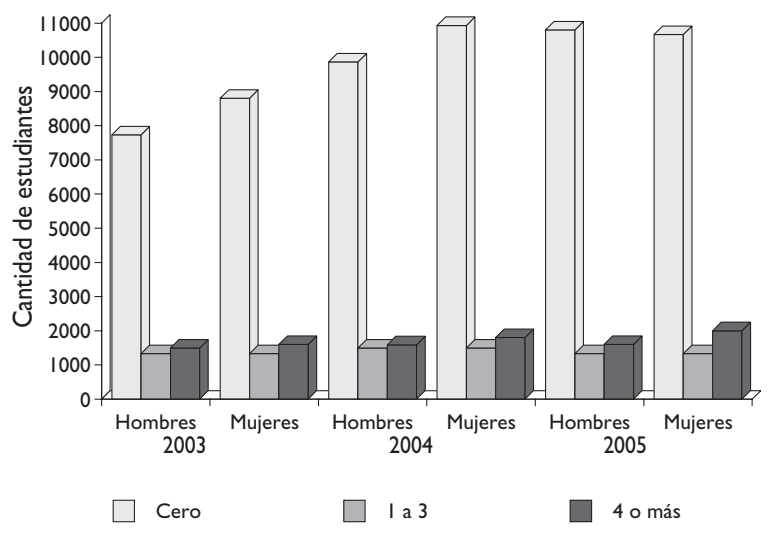

Fuente: Examen Médico Automatizado. Dirección General de Servicios Médicos. Universidad Nacional Autónoma de México. Batería índice CPO. Reportes ecológicos Nivel Medio Superior, 2003, 2004 y 2005; $n=77$ I9| estudiantes

Figura 2. Distribución porcentual de pérdida dental POR GENERACIÓN Y SEXo. MÉXICO, DF

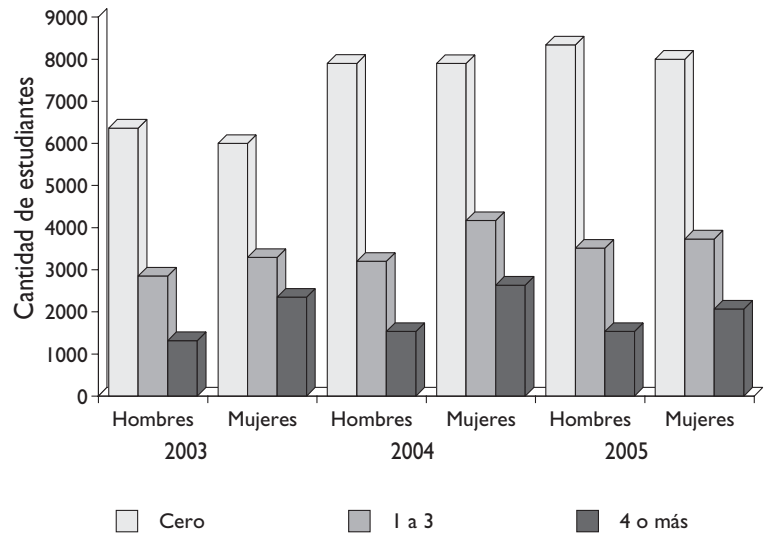

Fuente: Examen Médico Automatizado. Dirección General de Servicios Médicos. Universidad Nacional Autónoma de México. Batería índice CPO. Reportes ecológicos Nivel Medio Superior, 2003, 2004 y 2005; $n=77$ I9I estudiantes

Figura 3. Distribución Porcentual de dientes obtURADOS POR GENERACIÓN Y SEXO. MÉXICO, DF

similar para el número de dientes obturados (100 810). En relación con el número de dientes perdidos (155 834), la razón fue mayor (2:1).

Para calcular las necesidades de tratamiento se consideró el número de dientes cariados, el de piezas perdidas y el de dientes obturados de la población de estudio. Con base en ello, se dividió el número de dientes cariados entre el resultado de la sumatoria del CPO (356 887) y se obtuvo como resultado una necesidad de tratamiento de al menos un diente cariado o perdido para cada estudiante.

\section{Discusión}

La prevalencia de caries de $48 \%$ que se encontró en la población de estudio, que comprende las edades de 15 a 18 años, fue menor a la de 58\% informada en el plano nacional y a la de $88.6 \%$ identificada para el Distrito Federal en la población de 15 años de edad. ${ }^{14}$ Cabe resaltar que $28.1 \%$ de los estudiantes rebasa la meta propuesta por la OMS ( $\leq 3$ dientes con caries). Los datos obtenidos muestran una diferencia de $40 \%$ menos respecto de los datos publicados para el Distrito Federal, situación que puede explicarse debido a las características propias de la población de ingreso a la universidad, alumnos procedentes del Distrito Federal y el Estado de México. Por lo anterior, es posible inferir que la educación es un factor predictivo para la caries dental, en concordancia con otros estudios en los que se ha demostrado la influencia de los factores sociales como determinantes para la presencia de este problema. 22,23

salud pública de méxico / vol. 50, no. 3, mayo-junio de 2008 
Cabe señalar que la prevalencia de dientes cariados es similar a la de dientes obturados durante todo el periodo del estudio.

En lo referente a pérdida dental se encontró una prevalencia de $23.8 \%$, porcentaje mayor al comunicado en la investigación realizada en adolescentes que solicitaron atención odontológica en la Facultad de Odontología durante el año 2006 (19.1\%). ${ }^{17}$ Es importante resaltar que en la bibliografía revisada se encontraron escuetas referencias relacionadas con este factor.

Dentro de los resultados encontrados, el porcentaje de personas que conservan todos los dientes fue de $65.8 \%$, resultado que al compararse con los objetivos de la OMS $(85 \%)$, arroja un porcentaje inferior. ${ }^{12} \mathrm{Si}$ se considera la razón de dientes perdidos por estudiante, puede advertirse, por lo tanto, que cada estudiante sufriría la ausencia de cuando menos dos dientes.

La experiencia de caries en los estudiantes (índice CPOD) fue de 5, lo cual representa un número mayor al observado para la población nacional de 15 años (1.9) pero similar al del DF (5.3), concordancia que se puede inferir por el tipo de población estudiada.

Por último, si se toma en cuenta la prevalencia de caries, es posible deducir que $48 \%$ de la población requiere tratamiento odontológico, con la atención de al menos un diente.

\section{Conclusiones}

Los resultados del estudio permiten concluir que a pesar de que se reconoció un porcentaje menor respecto de lo informado en los planos nacional y regional, la prevalencia de caries en casi la mitad de la población es todavía un problema de salud bucal de importancia, si se considera la trayectoria natural de este padecimiento como factor de riesgo para la pérdida dental y sus implicaciones en la calidad de vida de las personas.

En relación con la pérdida dental, la razón de dos dientes perdidos por estudiante pone de manifiesto la necesidad de instrumentar políticas que promuevan condiciones ambientales para facilitar y fortalecer una cultura de prevención y promoción a la salud (prácticas de autocuidado y estilos de vida), que integren la salud bucal dentro de la salud general para conseguir mejores resultados.

En México son pocos los estudios que incluyen a estudiantes mayores de 12 años que permitan precisar las necesidades de tratamiento a partir de la descripción epidemiológica de los problemas en la salud bucal. Por consiguiente, con los hallazgos obtenidos se pueden diseñar programas de atención específicos de acuerdo con las necesidades observadas y se justifica la relevancia de realizar estudios orientados a la evaluación y efecto de tales intervenciones.

La proporción de necesidades de tratamiento indica que cada estudiante requiere la atención de por lo menos un diente; esto, si se analiza de manera individual, pareciera un problema mínimo, pero al analizar las necesidades de atención dental de este grupo poblacional el panorama cambia. Al tomar en cuenta que el costo del tratamiento más económico en la Facultad de Odontología de la UNAM para una caries dental es de 60 pesos mexicanos, los recursos económicos para atender este problema ascenderían a 6014580 pesos. Asimismo, con base en el costo cotizado por esta institución para el reemplazo de los dientes faltantes, el gasto representaría una cantidad de 31026800 pesos.

Los resultados evidencian la necesidad de contar con información del estado de salud bucal de toda la población y no restringir los datos epidemiológicos a la población considerada como de alta vulnerabilidad. De igual forma, un aspecto esencial para mejorar la salud de la población es contar tanto con información que permita conocer la distribución de las alteraciones como la relación de factores de riesgo, por lo que es importante diseñar subsecuentes investigaciones para identificar el modo en que se modificaron en este grupo poblacional los hábitos nocivos adoptados durante su estancia en la institución, como factores de riesgo en el desarrollo de este padecimiento. Los estilos de vida en los estudiantes que ingresan al nivel medio y medio superior se alteran por la gran vulnerabilidad que representa el estado de salud que predomina, debido al proceso de socialización al que se enfrentan y la accesibilidad a diversos hábitos dietéticos (frecuencia y acceso a comida chatarra) y adictivos (consumo de alcohol, drogas, tabaco, entre otros), los cuales son determinantes para el desarrollo de enfermedades sistémicas y bucodentales.

Por último, para mejorar la calidad de vida de los estudiantes es fundamental impulsar políticas y medidas de salud bucal, integradas dentro de programas nacionales e institucionales. Cabe mencionar que reconocer la importancia de la salud bucal en la salud general constituye el ingrediente principal de cualquier programa nacional de salud.

\section{Referencias}

I. Irigoyen ME, Zepeda MA, Sánchez TL, Molina N. Prevalencia e incidencia de caries dental y hábitos de higiene bucal en un grupo de escolares del sur de la Ciudad de México: estudio de seguimiento longitudinal. Rev ADM 200I;52(3):98-104. 
2. Maupome G.An introspective qualitative report on dietary patterns and elevated levels of dental decay in a deprived urban population in northern México.ASDC J Dent Child 1998;65:276-285.

3.Velásquez O,Vera H, Irigoyen ME, Mejía A, Sánchez TL. Cambios en la prevalencia de la caries dental en escolares de tres regiones de México: encuestas de 1987-1988 y de 1997-1998. Rev Panam Salud Publica 2003;13(5):I-14.

4. Mazariegos ML,Vera $H$,Velázquez O. Intervención de la Secretaría de Salud en salud bucal. Bol Med Hosp Infant Mex 2003;60(2):225-234.

5. Rivera G, Martínez J, Hernández E. Caries dental e higiene bucal en adolescentes. Rev ADM 2006;52(6):23I-234.

6. Moreno A, Carreón J,Alvear G, López S, Vega L. Riesgo de caries en escolares de escuelas oficiales de la Ciudad de México. Rev Mex Pediatr 200I;68(6):228-233.

7.Tascón JE, Cabrera GA. Creencias sobre caries e higiene oral en adolescentes del Valle del Cauca. Colombia Med 2005;36:73-78.

8. Novales XJ, Cancino O, Oropeza MV, Siciliano M,Valdivia SY, Rosas G, et al. Indicadores de salud bucal en alumnos de secundaria de un área metropolitana de la Ciudad de México. Rev Mex Pediatr 2003;70(5): 237-242.

9. Medina CE, Maupomé G,Avila L, Pérez R, Pelcastre B, Pontigo AP. Políticas de salud bucal en México: disminuir las principales enfermedades. Una descripción. Rev Biomed 2006; 17:269-286.

10. Carvalho JC, D'Hoore W,Van Nieuwenhuysen JP. Caries decline in the primary dentition of belgian children over 15 years. Community Dent Oral Epidemiol 2004;32:277-282.

II. Federation Dentaire Internationale. Goals for oral health in the year 2000. Newsletter 1982;March:5-8.

12. Hobdell M, Petersen PE, Clarkson J. Goals for oral health 2020. Int Dent J 2003;53:285-288.

13. Secretaría de Salud. Programa Nacional de Salud 200 I-2006. Estrategia: reducir los rezagos en salud que afectan a los pobres. Programa de Acción: Salud Bucal. México: SSA, 200I:97.
14. Centro Nacional de Vigilancia Epidemiológica y Control de Enfermedades. Encuesta Nacional Caries Dental 200I. México, DF: Programa de Salud Bucal, 200I.

15. Maupomé G. Prevalencia de caries en zonas rurales y periurbanas marginadas. Salud Publica Mex 1993;35(4):357-367.

16. Rivas J, Salas MA, Treviño M. Diagnóstico situacional de las afecciones bucodentales en la población de la ciudad de Zacatecas, México. Rev ADM 2000;52(6):218-221.

17. Pérez A. Estudio comparativo de las principales causas de pérdida dental en los alumnos de nuevo ingreso a nivel medio superior y superior, mediante el EMA. (2004-2005) (2005-2006). México, DF: Universidad Nacional Autónoma de México, 2007.

18. Jun A, Yuichi A, Rahena A, Hitoshi A, Mineo M, Manabu M. Reasons for permanent tooth extractions in Japan.J Epidemiol 2006;16:214-219.

19. Pineda M, Castro A, Watanabe R, Chein S,Ventocilla M. Necesidades de tratamiento para caries dental en escolares de zonas urbano y urbano marginal de Lima. Odontol Sanmar 2000; I (6):26-32.

20. López J], González de Cossío OM. Examen Médico Automatizado, ISBN 970-32-1770-2. Libro electrónico DGSM-UNAM. México: Programa Universitario de Investigación en Salud (PUIS), 2002.

21.Título Quinto: Investigación para la salud. Diario Oficial de la Federación 1984. Última reforma 19 de junio 2006. México: Secretaría de Gobernación, 2006.

22. Pérez SA, Gutiérrez MP, Soto L,Vallejos AA, Casanova JF. Caries dental en primeros molares permanentes y factores socioeconómicos en escolares de Campeche, México. Rev Cubana Estomatol 2002;39(3): 265-28I.

23. Romo MR, Herrera MIJ, Bribiesca ME, Rubio J, Hernández MS, Murieta JF. Caries dental y algunos factores sociales en escolares de Cd. Nezahualcoyotl. Bol Med Hosp Infant 2005;65(2): I 24-I35. 